\title{
Workshop on Lunar Calibration for Satellite Remote Sensing
}

\author{
C. E. Cramer \\ National Institute of Standards and Technology, \\ Gaithersburg, MD 20899 \\ claire.cramer@nist.gov
}

Tracking climate variables at the levels of precision and accuracy required to detect global change requires satellite sensors to make highly consistent measurements that can be compared to measurements made at different times and with different instruments. Gaps in climate data records, such as those resulting from launch delay or instrument failure, and inconsistencies in radiometric scales between satellites can introduce unnecessary measurement error and thus undermine the credibility of fundamental climate data records. To address these issues, leading experts in satellite remote sensing and lunar observation and modeling assembled at the National Institute of Standards and Technology from 12-15 May 2012 for a workshop to discuss the utility of and strategies for using the Moon to calibrate satellite remote sensing measurements. This report summarizes the outcome of the workshop, including suggested steps to maximize the value of the Moon as an exoatmospheric calibration source for satellite remote sensing.

Key words: calibration; lunar spectral irradiance; moon; radiometry; remote sensing.

Accepted: June 29, 2016

Published: July 11, 2016

http://dx.doi.org/10.6028/jres.121.016

\section{Introduction}

A workshop was held at the National Institute of Standards and Technology from 12 to 15 May 2012 to discuss the utility of the Moon in calibrating satellite sensor observations. Over 50 participants from government, academia, and the private sector came together to assess the potential benefits of establishing the Moon as a calibration standard traceable to the International System of Units (SI) and identify scientific programs to establish lunar calibration techniques capable of meeting present and future calibration needs. Participating government agencies included the National Aeronautics and Space Administration (NASA), National Geospatial-Intelligence Agency (NGA), National Institute of Standards and Technology (NIST), National Oceanographic and Atmospheric Administration (NOAA), United States Geological Survey (USGS), and the United States Naval Observatory (USNO).

Technical presentations at the workshop surveyed current use of the Moon as a radiometric calibration standard, ground-based observations of the Moon, atmospheric monitoring for ground-based observations, above-the-atmosphere observation platforms, and calibration needs for future missions. The presentations and discussion focused primarily on satellite remote sensing at reflected solar wavelengths, $380 \mathrm{~nm}$ to $2500 \mathrm{~nm}$. Applications in atmospheric science and related work in astronomy also were discussed. The workshop concluded with a discussion that generated suggestions for exploiting the Moon's potential as a radiometric calibration source.

\section{Use of Lunar Calibration in Satellite Remote Sensing}

Comparing lunar views with a reliable model that predicts the Moon's spectral irradiance as a function of Sun-Moon-Observer geometry has many advantages for the calibration of satellite sensors. The Moon is 
an exceptionally stable reflector that does not degrade in time like lamps or solar diffusers [1], nor does it require constant maintenance like the precisely-calibrated Earth surface sites used for vicarious calibration [2]. Lunar views can use the full optical path of the sensor. The Moon is in a dynamic range similar to Earth scenes and is accessible to satellites in any Earth orbit. Lunar calibration provides an avenue for instrument cross-comparison that does not require both instruments to view the same target at the same time. Finally, calibrations made using the Moon can become more accurate and more precise over time as more lunar views are acquired with an instrument, and as lunar models improve and existing data are reprocessed with the improved models.

If the model of the Moon's spectral irradiance is accurate as well as precise, ideally over a broad range of lunar phase and libration angles, lunar calibration can be used to determine changes in sensor response on transfer to orbit, eliminating the current bottleneck in verifying absolute calibration post-launch. If operational systems could achieve accurate on-orbit calibration with 3 to 5 measurements rather than the more typical 30 to 50, the fraction of immediately useful data out of an operational mission could increase by as much as $40 \%^{1}$. An accurate lunar model would also allow lunar views to act as a tie point for intersensor comparisons even if there are gaps in the collection period resulting from, for example, launch delays or instrument failure. Comparisons to data from instruments that are no longer operational can also be made, provided those previous instruments have acquired lunar images. One particularly important application is the potential ability to connect climate data records derived from data taken before and after a gap in satellite coverage.

Thus far, lunar views have been used primarily for tracking temporal changes in the radiometric response of satellite sensors. The canonical lunar calibration success story is the Sea-Viewing Wide Field of View Sensor (SeaWiFS), which achieved $0.1 \%$ relative radiometric stability trending by comparing monthly lunar views with a model developed by USGS that predicts lunar spectral irradiance as a function of Sun-Moon-Observer geometry [3]. Other Earth-observing instruments, such as the Moderate Resolution Imaging Spectrometers (MODIS) on NASA's Aqua and Terra satellites, the Hyperion hyperspectral imaging spectrometer on NASA's Earth Observing-1 satellite, and the Multi-Angle Imaging SpectroRadiometer (MISR) on Terra have also used the Moon as a calibration source. Lunar calibrations are planned for the Advanced Baseline Imager (ABI) on the joint NOAA/NASA Geostationary Operational Environmental Satellites - R Series (GOES-R), Landsat 8, and the Visible Infrared Imagine Radiometer Suite (VIIRS) on the Suomi National Polar-orbiting Partnership (NPP) satellite. In addition to radiometric response, the Moon has proved useful for characterizing various other sensor properties, including stray light, ghosting, instrumental modulation transfer functions, focal plane to focal plane comparisons, band to band registrations, focal plane temperature, and the effect of scan angle on detector response ${ }^{2}$.

The USGS model used for relative radiometric calibration is an analytic model with continuous variables of phase and libration [4]. It is based on a series of lunar observations made in 32 spectral passbands with a nominal width of $10 \mathrm{~nm}$ and spanning a spectral range of $350 \mathrm{~nm}$ to $2350 \mathrm{~nm}$. The eightyear series of observations provides good coverage of the phase and libration parameter space. As SeaWiFS has demonstrated, the USGS model can predict changes in lunar spectral irradiance at a given lunar phase and within a given passband at the sub-percent level. A recent comparison of data from SeaWiFS and MODIS suggests that residual phase dependence in the USGS model is below $2 \%$ over a phase angle range of $-80^{\circ}$ to $-6^{\circ}$ and $+5^{\circ}$ to $82^{\circ}$ [5]. The magnitude of wavelength-dependent systematic effects, which depends on the scheme used to translate the USGS model to the passbands used by the instrument in question, is likely to be at a similar level. The absolute calibration of the USGS model is not SI-traceable and is thought to be accurate at the 5 to $10 \%$ level.

Workshop participants stressed that mission scientists may be hesitant to commit to a program of lunar views until the absolute scale of the lunar model as well as phase, libration, and wavelength-dependent systematics are demonstrated to be below their mission's on-orbit radiometric calibration requirements, especially if a spacecraft maneuver is required to view the Moon. Measurements to establish an SItraceable absolute scale, better quantify phase- and libration-dependent model uncertainties, and improve

\footnotetext{
${ }^{1}$ Establishing values of sensor calibration coefficients through a series of 30-50 observations of a vicarious calibration target can take as long as two years of a five-year mission.

${ }^{2}$ Presented by R. E. Eplee at this workshop.
} 
spectral resolution and wavelength coverage will therefore be doubly beneficial: past and present lunar calibrations will be improved and adoption of lunar calibration methods will increase, leading to a larger set of data records with linked calibrations.

Finally, participants noted that despite its many advantages, the Moon will not solve every calibration problem. Each instrument will require different strategies for integrating images of the resolved lunar disk and correcting for geometric distortion. The Moon does not cover the full dynamic range of Earth scenes captured by satellite sensors. Reflected light from the Moon is slightly polarized. Solar variability will affect the most precise measurements, especially at short wavelengths. Usability depends on phase angle. Many missions need to calibrate more often than is either practical or possible to view the Moon. For these reasons and others, lunar calibration is best used together with complementary techniques.

\section{Establishing the Moon as an SI-Traceable Standard}

Although specific calibration requirements vary from mission to mission, there was a consensus among workshop participants that developing an improved lunar spectral irradiance model with an SI-traceable calibration, continuous wavelength coverage with a resolution of a few $\mathrm{nm}$, and $k=1$ uncertainties at or below $1 \%$ would be broadly beneficial.

Preliminary work at NIST indicates that ground-based observations with a calibrated telescope and spectrograph may be capable of making SI-traceable measurements of the Moon's spectral irradiance with sub-percent accuracy at visible wavelengths [6]. The NIST technique provides continuous spectral coverage but is limited to stable, isotropic atmospheric conditions and wavelengths unaffected by molecular absorption. Water vapor absorption limits the accuracy of ground-based calibrations at wavelengths longer than $\approx 900 \mathrm{~nm}$.

The first limitation, a stable, isotropic atmosphere, can be addressed through direct measurements of atmospheric extinction co-located and contemporaneous with the lunar observations. The multi-wavelength Astronomical Lidar for Extinction under development at the University of New Mexico was presented as one possible solution that would complement the capabilities of NIST's lunar telescope.

The second limitation, water vapor absorption, is best addressed by moving the observing platform to an altitude at which atmospheric water vapor absorption is lower than the desired measurement uncertainty. A satellite-based platform would allow a long time series of measurements devoid of atmospheric extinction but presumes a solution to the on-orbit calibration problems the improved lunar calibrations are meant to solve. Aircraft and balloon-based platforms provide an intermediate option, flying above $99 \%$ of the Earth's atmosphere while allowing pre- and post-flight laboratory calibration. Presentations at the workshop outlined capabilities of Global Hawk and ER-2 aircraft ${ }^{3}$, and stratospheric balloons.

\section{The Path Forward}

Workshop participants agreed that it is important to maintain current lunar calibration capabilities in addition to addressing shortcomings in the existing lunar model. The group generated the following list of suggestions for making the most of current capabilities, logical next steps, and longer-term prospects:

1. Widespread use of the Moon as a calibration standard is hampered by the lack of stable funding for maintenance, further development, and distribution of the USGS model. Since its publication in 2005, the model has been adapted for a wide variety of applications on an ad hoc basis. A webbased interface or software package, with version control and documentation, capable of adapting the model for use with different spectral ranges, spectral resolution, and Sun-Moon-Observer geometries would greatly enhance the model's utility. The complications of providing a web

\footnotetext{
${ }^{3}$ Certain commercial equipment, instruments, or materials are identified in this paper to specify the information presented at the workshop adequately. Such identification is not intended to imply recommendation or endorsement by the National Institute of Standards and Technology, nor is it intended to imply that the materials or equipment identified are necessarily the best available for the purpose.
} 
service that generates calibration data with regard to International Traffic in Arms (ITAR) restrictions on satellite enhancements were discussed.

2. A series of SI-traceable ground-based measurements of lunar spectral irradiance at visible wavelengths with continuous spectral coverage and spectral resolution of a few nm is straightforward, inexpensive compared to the satellite missions to be calibrated, and capable of addressing the primary limitations of the USGS model. At an approximate cost of no more than a few million dollars per year, with a total project cost of roughly $\$ 10$ million, an automated observatory tracking the Moon whenever it is observable could provide data spanning an adequate range of phase and libration angles in 3 to 5 years. To maximize data quality, the observatory should be located at a high altitude site with good atmospheric conditions and a suite of atmospheric monitoring instrumentation, such as NOAA's Mauna Loa Observatory. Atmospheric corrections could be validated with balloon or aircraft measurements. Remote sensing data with corresponding lunar views could be back-calibrated when the improved model becomes available.

3. Satellite-based measurements of the Moon would be a valuable complement to ground-based measurements, particularly at infrared and ultraviolet wavelengths. While establishing an SItraceable absolute calibration from a satellite-based platform remains a challenge, a dedicated satellite instrument with good trending, good relative spectral responsivity calibration, and continuous spectral coverage would be ideal for exploring possible systematic effects in the USGS model.

4. Although the USGS model has been used primarily for spectral irradiance, the Moon's radiance is also a useful quantity. Bringing radiance model development along with other programs would benefit a number of specific Department of Defense programs, as would the longer-term objective of developing radiance and reflectance models with high spatial resolution.

5. Models of lunar spectral irradiance and radiance as well as a characterization of surface nonuniformity in the thermal infrared are also of interest. The Moon is both an emitter and a reflector in this wavelength range, and exhibits large temperature variations on short spatial scales. For these reasons as well as the need to measure from above the Earth's atmosphere, instrument design and data analysis are more challenging than for the reflected solar wavelength range discussed above.

Given the broad range of applications and considerable benefit to satellite programs across the government, it would be appropriate for government agencies to take the lead in implementing the solutions outlined above. To take advantage of each agency's area of expertise while ensuring that diverse needs are met, a lunar calibration program would best be implemented through a strong collaboration between NASA, NIST, NOAA, and USGS. NIST, as the government agency responsible for maintaining and developing standards, would be a logical coordinating agency, with each cooperating agency taking the lead on activities in their areas of expertise.

\section{Conclusions and Outlook}

At present, the Moon is an essential piece of the overall sensor characterization and calibration process for a wide variety of remote sensing applications. Further development of the Moon as a radiometric calibration standard will benefit future programs while adding value to present and past data sets containing lunar views. Specifically, an SI-traceable calibration of lunar spectral irradiance at reflected solar wavelengths with an uncertainty of $1 \%$ and continuous spectral coverage, combined with a model such as the USGS model mentioned above, would meet the needs of many existing and planned programs, and is achievable with a series of ground- and space-based measurements. In the interim, it is critical not to let current capabilities lapse as new activities are pursued.

\section{References}

[1] Kieffer HH (1997) Photometric Stability of the Lunar Surface. Icarus 130:323-327. http://dx.doi.org/10.1006/icar.1997.5822 
[2] Clark DK, Yarbrough MA, Feinholz ME, Flora S, Broenkow W, Kim YS, Johnson BC, Brown SW, Yuen M, Mueller JL (2003) MOBY, a radiometric buoy for performance monitoring and vicarious calibration of satellite ocean color sensors: Measurement and data analysis protocols. Ocean Optics Protocols for Satellite Ocean Color Sensor Validation, Revision 4, Volume VI: Special Topics in Ocean Optics Protocols and Appendices, ed Mueller JL, Fargion GS, McClain CR (NASA Goddard Space Flight Center,Greenbelt, MD), NASA/TM2003-211621/Rev4-Vol.VI:3-34.

[3] Barnes RA, Eplee RE, Patt FS, Kieffer HH, Stone TC, Meister G, Butler JJ, McClain CR (2004) Comparison of SeaWiFS measurements of the Moon with the U.S. Geological Survey lunar model. Appl Opt 43(31):5838-5854. http://dx.doi.org/10.1364/AO.43.005838

[4] Hugh HK, Thomas CS (2005) The Spectral Irradiance of the Moon. The Astronomical Journal 129(6):2887. http://dx.doi.org/10.1086/430185

[5] Eplee JRE, Sun J-Q, Meister G, Patt FS, Xiong X, McClain CR (2011) Cross calibration of SeaWiFS and MODIS using onorbit observations of the Moon. Appl Opt 50(2):120-133. http://dx.doi.org/10.1364/AO.50.000120

[6] Subsequently published as Cramer CE, Lykke KR, Woodward JT, Smith AW (2013) Precise Measurement of Lunar Spectral Irradiance at Visible Wavelengths. J Res Natl Inst Stan 118:396-402. http://dx.doi.org/10.6028/jres.118.020

About the author: C. E. Cramer was a physicist in the Sensor Science Division at NIST and was the principal organizer of this workshop. The National Institute of Standards and Technology is an agency of the U.S. Department of Commerce. 